\title{
Optical MEMS based on Silicon-on-Insulator (SOI) for Monolithic Microoptics
}

\author{
Wilfried Noell, Winston Sun, and Nico de Rooij \\ University of Neuchâtel, Institute of Microtechnology (IMT), Rue Jaquet Droz 1, CH-2007 Neuchâtel, Switzerland, \\ phone: +41-32-720-5548, fax: +41-32-720-5711,e-mail: Wilfried.Noell@unine.ch \\ Hans Peter Herzig, Omar Manzardo, and René Dändliker \\ University of Neuchâtel, Institute of Microtechnology (IMT), Rue Breguet 2, CH-2000 Neuchâtel, Switzerland
}

\begin{abstract}
Microelectromechanical systems (MEMS) combined with optical components add optical functionality to devices and led to the terms Optical MEMS or MOEMS. The underlying technology of the presented devices is the silicon-on-insulator (SOI) based batch fabrication, which delivers small, reliable and lasting monolithic bulk silicon structures for commercial devices with the advantage of being very insensitive to temperature changes. The particular strength of the technology are monolithic horizontal and vertical micromirrors for a variety of applications.
\end{abstract}

Key Words: Microstructure devices; Couplers, switches, and multiplexers; Mirrors; Optomechanics;

\section{Introduction}

The significant influence of micro-electro mechanical systems (MEMS) in modern technology has led to many new and very small and well-controlled devices. In the optical domain MEMS components are becoming more and more relevant, particularly for the telecommunication industry and optical sensor technology [1].

In telecommunication industry are four main device domains: Amplifiers, switches or optical cross connectors (OXC), filters and variable optical attenuators (VOA). While optically pumped fiber amplifiers do not require any mechanical components, OXC, VOA, and filters are, however, based on mechanically or thermally driven elements and are hence relatively slowly working devices, which is widely accepted because of their use in slowly working routers and equalizers.

Besides for telecommunication components, MOEMS devices are also used in analysis instruments such as optical spectroscopes and they can reduce the size of such instruments considerably. Our step towards this optical domain is the MEMS based Fourier transform spectrometer (FTS).

\section{SOI Technology}

The IMT silicon bulk micromaching is based on $100 \mathrm{~mm}$ SOI wafers, which comprise a handle wafer as a base, a buried $\mathrm{SiO}_{2}$ layer (1 to $2 \mu \mathrm{m}$ thick), and a device layer $(10$ to $100 \mu \mathrm{m})$ on top made of single crystalline silicon (Fig. 1). It is an extremely simple technology with very few process steps, where deep reactive ion etching (DRIE) is utilized to pattern the device layer and the handle wafer. DRIE can create almost arbitrary 3D shapes. The SOI bulk micromachining is very different from both surface micromaching, which utilizes polycrystalline silicon as a device layer, and classical bulk micromachining based on $\mathrm{KOH}$ etching, where silicon crystal planes constrain the patterning considerably. The

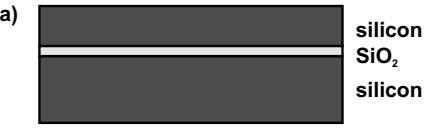

c)

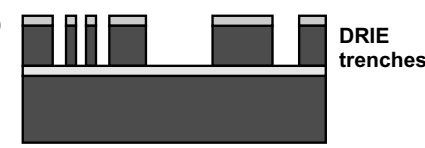

e)

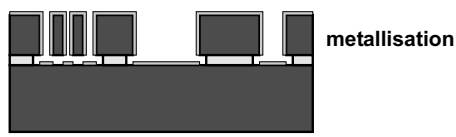

b)

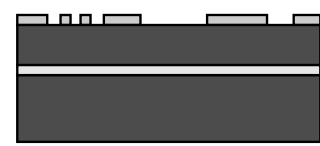

d)

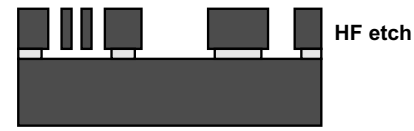

f)

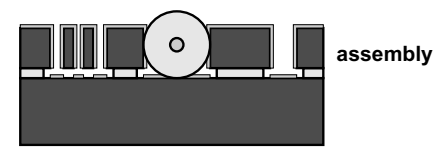

Fig. 1. SOI technology: a) The SOI wafer is a composite of a silicon substrate wafer, a buried $\mathrm{SiO}_{2}$ layer, and a device layer. b) and c) The wafer is patterned by DRIE utilizing a photoresist mask. d) The structures are released by wet-etching the $\mathrm{SiO}_{2}$. e) The metallisation improves the surface conductivity and reflectivity. f) Integrated alignment structures assist in the assembly of $\mu$-optical components. DRIE-patterned surfaces can be made optically flat on top and sidewalls and the structures have excellent mechanical properties and show no fatigue. Due to the thickness of the structures, coatings and metallization of the devices have very little or no influence on the shape of the structures. The DRIE also allows producing highly accurate U-shaped guiding structures for optical components such as optical fibers. The pre-alignment is in the sub- $\mu \mathrm{m}$ range. 


\section{Micromirrors for Optical Switches (OXC) and Fourier Transform Spectrometers (FTS)}

The first SOI development was a $2 \times 2$ fiber $O X C$, which is now commercially available via the company Sercalo. The $2 \times 2$ OXC has two input and two output fibers [2]. The four fibers sit in Ugrooves and are oriented at right angles to each other. Their endfaces all meet at one point, where an extremely smooth and movable gold-coated mirror is located. In a further development a $1 \times 4$ switch was microfabricated, which has 1 input and 4 output fibers. In both cases, the mirrors are attached to a beam that is electrostatically actuated by comb drives (Fig. 2a).

The optical loss in the switch is minimized by completely surrounding the optical components with refractive index matching fluid, which reduces both the refractive index step and the beam divergence in between the fibers. In the case of the $2 \times 2$ OXC, the insertion loss is less than $0.5 \mathrm{~dB}$, the cross talk as low as $-70 \mathrm{~dB}$, and the switching time $500 \mu \mathrm{s}$. The live time was tested to be $5 \cdot 10^{9}$ cycles at $85^{\circ} \mathrm{C}$.

FTS is a well-known technique to measure the spectra of a weak and extended light source whereas it offers a high signal-tonoise ratio. The MOEMS FTS utilizes the commonly used Michelson interferometer configuration with a scanning $\mu$-mirror and occupies a total footprint of only $5 \times 4 \mathrm{~mm}^{2}$ (Fig. 2b) [3] While the first approach was based on an external beam splitter, we are currently investigated different integration technologies. The optical path difference reached $77 \mu \mathrm{m}$ and gained an optical linewidth for a He-Ne laser $(\lambda=632.8 \mathrm{~nm})$ of about $6 \mathrm{~nm}$.

\section{Variable Optical Attenuator (VOA)}

Classically, VOA's are based on a light-shutter configuration that varies the optical intensity between two opposing fibers or collimators. In a novel joined approach we are fabricating a VOA based on a set of $16 \mu$-mirrors (Fig. 2c) [4]. The size of the mirrors is chosen such that each mirror reflects the intensity of one bit, yielding 16 intensity levels, which corresponds to 16 bit. Thus the light intensity is digitally modulated, rather than continual and highly non-linear as it is the case with shutters.

\section{Acknowledgement}

The authors like to thank Cornel Marxer from Sercalo Ltd. for his great support and development in the area of the SOI technology. We are particularly grateful to N. Riza (Nuonics Inc., Oralando, FL, U.S.A.) in the joint collaboration of the digital VOA. The $1 \times 4$ switch is a very fruitful collaboration with the Singapore Institute of Manufacturing Technology (SIMTech), who we also like to thank for their financial support. Additional financial support comes from the Croucher Foundation of Hong Kong, who we like to thank for their generosity.
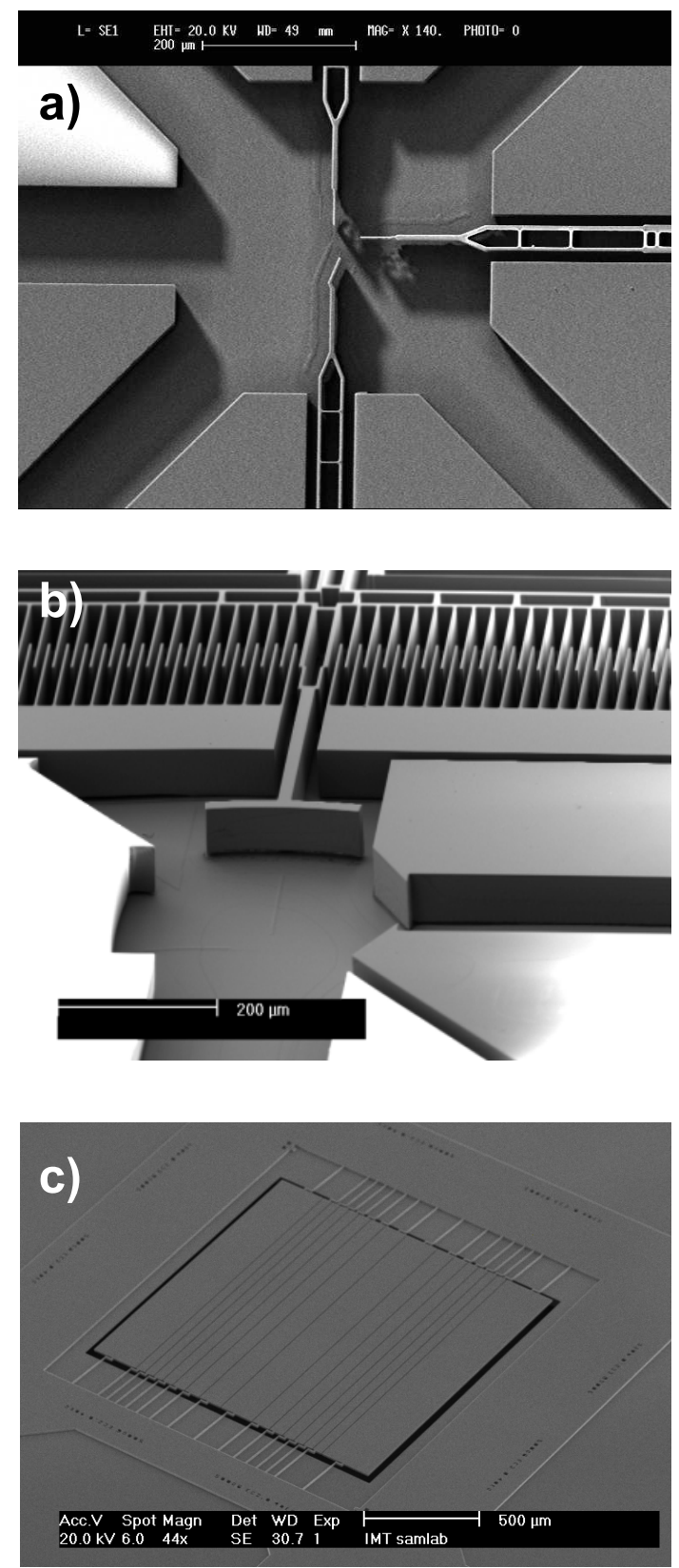

Fig. 2. Micromirrors: a) For an $1 \times 4$ optical cross connectors (OXC) each mirror is highly accurately pre-aligned with an optical fiber via U-grooves. b) In the FTS (Michelson interferometer) the scanning mirror also collimates the light in one dimension to increase the coupling efficiency between the in- and output fibers [3]. c) In a new joint approach of a VOA each of the $16 \mu$-mirrors represents one bit of a 16 bit intensity modulation [4].

\section{References}

[1] W. Noell, P.-A. Clerc, L. Dellmann, B. Guldimann, H.P. Herzig, O. Manzardo, C. Marxer, K. Weible, R. Dändliker, N. de Rooij, "Applications of SOI-based optical MEMS", J. of Selected. Topics of Quant. Electr. March (2002).

[2] C. Marxer and N.F. de Rooij, "Micro-optomechanical 2 x 2 switch for single-mode fibers based on a plasma-etched silicon mirror and electrostatic actuation", J. of Lightwave Technology 17, 2-6 (1999).

[3] O. Manzardo, H.-P. Herzig, C.R. Marxer, N.F. de Rooij, "Miniaturized time-scanning Fourier Transform Spectrometer based on silicon technology", Opt. Lett. 24, 1705-1707 (1999).

[4] Riza N.A. and Sumriddetchkajorn S., " Digitally controlled fault tolerant multiwavelength programmable fiber-optic attenuator using a two dimensional digital micromirror devices," Optics Letters, 24, March 1, 1999, pp. 282-284. 